$45 \mid 2010$

Langue française, identité(s) et école(s) : le cas de la minorité catholique au Levant (milieu XIX ${ }^{\mathrm{e}}-\mathrm{XX}^{\mathrm{e}}$

siècles). Représentations identitaires et apprentissage des langues : Europe, bassin méditerranéen ( $\mathrm{XVI}{ }^{\mathrm{e}}-\mathrm{XX}{ }^{\mathrm{e}}$ siècles)

\title{
Une union éternelle? Les catholiques libanais et les écoles françaises au Liban 1900-1950
}

\section{Esther Moeller}

\section{(2) OpenEdition \\ $\checkmark$ Journals}

\section{Édition électronique}

URL : https://journals.openedition.org/dhfles/2413

DOI : $10.4000 /$ dhfles. 2413

ISSN : 2221-4038

Éditeur

Société Internationale pour l'Histoire du Français Langue Étrangère ou Seconde

\section{Édition imprimée}

Date de publication : 1 juin 2010

Pagination : 43-68

ISSN : 0992-7654

\section{Référence électronique}

Esther Moeller, « Une union éternelle ? Les catholiques libanais et les écoles françaises au Liban 1900-1950 », Documents pour l'histoire du français langue étrangère ou seconde [En ligne], 45 | 2010, mis en ligne le 13 mars 2014, consulté le 27 mai 2021. URL : http://journals.openedition.org/dhfles/2413 ; DOI : https://doi.org/10.4000/dhfles.2413

Ce document a été généré automatiquement le 27 mai 2021.

(C) SIHFLES 


\title{
Une union éternelle? Les catholiques libanais et les écoles françaises au Liban 1900-1950
}

\author{
Esther Moeller
}

\section{Introduction}

En 1844, l'archevêque maronite libanais, monseigneur Nicolas Murad, dédia sa Notice historique sur l'origine de la nation maronite et sur ses rapports avec la France au roi de France Louis-Philippe avec les mots suivants: «Permettez, Sire, que je place sous la protection de Votre Majesté une Euvre destinée à faire connaître et apprécier en France cette nation dévouée. $»^{1}$ En 2008, 160 ans plus tard, un ancien élève et professeur du collège catholique lazariste d'Antoura au Liban, Victor Hachem, publia un livre avec pour titre Le Français et nous. Dans ce livre, l'auteur explique que, selon lui, la langue française répugne au colonialisme ${ }^{2}$. Comment expliquer l'impression d'union éternelle entre les catholiques libanais et la France? Critiques et défenseurs de cette union sont unanimes à souligner la place cruciale des écoles françaises dans cette relation quasi mythique (Abou 1962 : 112). C'est pourquoi cette contribution traitera de la relation très particulière entre les catholiques libanais et les écoles françaises au Liban de 1900 à 1950. L'hypothèse à vérifier est que, jusque récemment, et malgré de légères tensions, le lien entre ces deux groupes est resté fort parce qu'il leur a servi à maintenir et justifier leur place au Liban.

2 Cette continuité est d'autant plus surprenante que les années 1900 à 1950 couvrent trois régimes politiques différents au Liban qui ont été fondamentaux pour la position à la fois des catholiques et des écoles françaises. Au Liban même, cette période correspond à la fin de l'Empire ottoman, en 1918, au mandat français de 1920 à 1943, et à l'indépendance du pays à partir de 1943. Bien qu'il ne soit donc pas juridiquement correct de parler de Liban avant 1920, cette appellation sera tout de même utilisée pour la période ottomane et désignera le mutasarifíya (province) du Ĝabal Lubnân / Mont 
Liban, ainsi que les villes côtières de Beyrouth, Tripoli et Saïda. En France, cette période fut marquée par la séparation de l'Église et de l'État en 1905, par les deux guerres mondiales, et par le passage de la III à la IV République.

3 Le plan de cette contribution suivra un ordre chronologique : alors que la période du régime ottoman témoignait d'une coopération étroite entre catholiques libanais et écoles françaises (pour des motifs religieux surtout), celle du régime mandataire fut marquée par un élargissement de cette coopération au profit d'un affermissement des deux groupes au sein de l'élite politique, économique et sociale du pays. La période d'indépendance vit finalement une continuation de cette relation étroite, mais aussi une plus grande ouverture vers d'autres communautés du pays, en particulier les sunnites.

4 Avant de procéder à l'analyse, il est important de définir brièvement ce qui sera entendu par catholiques libanais et écoles françaises au Liban. En ce qui concerne les catholiques, il y avait au Liban les Églises maronites, grecques-catholiques melkites, catholiques syriaques, chaldéennes, catholiques arméniennes et latines (Suermann 2010: 105-136). Contrairement aux autres Églises catholiques, les maronites constituaient une communauté très hétérogène, avec des paysans pauvres au nord du pays et une classe moyenne de mieux en mieux établie à Beyrouth. Parce que l'Église maronite constitue la communauté numériquement la plus importante, et que le patriarche maronite représente depuis le début du $\mathrm{XX}^{\mathrm{e}}$ siècle une figure politique influente ; que, de plus, ses membres ont massivement établi des liens avec la France et, par conséquent avec les écoles françaises, l'observation reposera sur cette communauté, bien que les autres communautés catholiques soient également prises en compte pour faire contrepoids à une image qui, sinon, serait trop homogène pour décrire une situation très complexe.

5 L'Église maronite repose sur la figure de l'ermite Maron qui vécut en Syrie au V $\mathrm{V}^{\mathrm{e}}$ siècle. Depuis les croisades, elle est unie avec Rome, tout en restant une Église orientale avec un patriarche qui réside au Liban. Ses membres vivaient et vivent principalement au Liban et en Syrie. À l'époque ottomane, ils constituaient une majorité au mutasarifiya du Gabal Lubnân / Mont Liban, dirigé depuis 1861 par un gouverneur chrétien, relativement autonome par rapport à la Sublime Porte (Traboulsi 2007 : 47-48). Dans les villes de la côte ils ne représentaient qu'une minorité, mais surtout à Beyrouth une minorité grandissante, puisque beaucoup de chrétiens quittaient les montagnes pour vivre dans les villes, en particulier à Beyrouth. Après le démantèlement de l'Empire ottoman, ce furent surtout les maronites qui s'engagèrent, représentés par le patriarche Elias Hoyek, dans les négociations de paix à Paris en 1919, pour la constitution d'un Liban autonome sous la protection de la France. D'autres communautés chrétiennes défendirent par contre l'idée d'une union du Liban avec la Syrie (Khoury 1993 : 181-187). Lors de l'instauration du mandat en 1920, les chrétiens, donc les catholiques, représentaient un peu plus de la moitié de la population. Le dernier recensement date de 1932, il n'y a donc pas de chiffres récents, mais il est très probable que les chrétiens ne constituent plus aujourd'hui la majorité du pays, dont la population a connu à la fois l'émigration de beaucoup d'entre eux et une immigration à majorité musulmane. Il est donc important de souligner que le Liban a constitué pendant longtemps le seul pays arabe abritant une grande population chrétienne et dont une communauté, les maronites, appartenait à l'élite politique, économique et sociale (Zamir $2000: 240$ ). 
6 Le terme d'écoles françaises désigne des institutions très diverses qui ont coexisté au Liban : à côté des écoles catholiques françaises, tenues par des ordres religieux, il y avait aussi des écoles de la Mission laïque française, de l'Alliance israélite universelle et, depuis les années 1920, un collège protestant français. Seules les écoles catholiques et les écoles de la Mission laïque française accueillaient un nombre important d'élèves catholiques, et l'analyse portera donc majoritairement sur ces deux types d'écoles.

7 Les archives disponibles étant principalement celles des écoles françaises en France et au Liban, et celles du ministère français des Affaires étrangères, la question sera surtout traitée à partir des écoles françaises. Mais le point de vue libanais pourra être intégré grâce à des mémoires et des entrevues avec d'anciens élèves libanais de ces écoles, ainsi que des articles de journaux. Une « histoire croisée » ainsi élaborée selon la conception de Michael Werner et Bénédicte Zimmermann fera comprendre que seul le croisement des perspectives françaises et libanaises permet d'avoir un jugement objectif sur la relation entre catholiques libanais et écoles françaises.

\section{La période ottomane : besoin et soutien réciproques}

8 La période ottomane se caractérise par le besoin et le soutien réciproques entre catholiques et écoles françaises. Bien que la fin de l'Empire ottoman fût marquée, du point de vue des influences extérieures, par la domination de la France, il ne faut pas oublier que cette suprématie n'était pas garantie d'avance. Au contraire, jusqu'en 1850, la langue italienne avait su s'imposer au Liban et la prépondérance du français après cette date demeurera toujours fragile (Abou 1962 : 188-189). Les missionnaires français n'étaient arrivés au Liban en grand nombre que dans la première moitié du XIX ${ }^{\mathrm{e}}$ siècle ${ }^{3}$. Leur but premier était de propager leur foi, à travers, entre autres, la fondation d'écoles. En même temps, étant donné qu'ils enseignaient le français et en français, les gouvernements français successifs les soutenaient massivement et les considéraient comme des agents parfaits pour diffuser la langue française contre les concurrences impériales, en premier lieu celles de la Grande-Bretagne et des États-Unis. Il est important de noter que les missionnaires français n'étaient pas venus au Liban de leur propre chef, mais parce que les catholiques du Liban les y avaient invités. On peut citer à ce sujet monseigneur Murad. Ce fait constitue une différence fondamentale avec d'autres régions sous influence française dont les habitants n'avaient jamais réclamé d'eux-mêmes une éducation française. Les liens des maronites avec l'Europe furent renforcés par la création d'un séminaire maronite à Rome en $1584^{4}$.

9 À partir de 1850, on constate un renforcement des institutions éducatives françaises au Liban. Un jalon important est constitué par la fondation de l'université Saint-Joseph à Beyrouth en 1875. Celle-ci servait différents groupes d'intérêt: les jésuites pour contrebalancer les restrictions qui les visaient en France, le gouvernement français qui faisait face à la concurrence américaine du Syrian Protestant College (Kassir $2003: 241$ ), et enfin les catholiques du Liban et du Proche-Orient qui pouvaient dès lors fréquenter une université officiellement catholique et dont les diplômes étaient reconnus en France (Awit \& Ducruet 2006: 39). Ce processus illustre aussi le décalage entre une jeunesse catholique libanaise très scolarisée et les autres communautés. Cela correspondait au système éducatif ottoman, dans lequel les différents groupes confessionnels fréquentaient souvent les écoles privées de leurs communautés dont peu seulement disposaient de moyens financiers importants, comme l'association 
sunnite de Beyrouth al-Maqâsid al-khairîya al-islâmîya. Il y avait aussi des écoles publiques, mais celles-ci étaient surtout fréquentées par les populations musulmanes (Fortna 2002: 55). Il y avait d'autre part des écoles catholiques locales, mais elles se révélaient moins attirantes, parce que les écoles françaises disposaient souvent de moyens financiers supérieurs. Pour les parents, à l'époque ottomane, bien connaître le français signifiait également augmenter les chances de leurs enfants d'obtenir un bon travail dans le commerce, dominé à l'époque par le marché français ${ }^{5}$. C'est précisément cette dernière raison qui incita des parents non catholiques à envoyer leurs enfants dans les écoles de la Mission laïque française, établie à Beyrouth depuis $1909^{6}$. En ce qui concerne les catholiques du Liban, l'enseignement français des écoles catholiques françaises comptait avant tout pour sa dimension chrétienne. Cette prédominance se révèle par exemple dans la lettre de Salîm Nasîf de Zahlé aux jésuites de Tanaïl, par laquelle l'auteur leur confiait un orphelin dont il était le tuteur, parce que depuis des générations, la famille de l'orphelin appartenait à la communauté catholique romaine ${ }^{7}$. De plus, les catholiques percevaient aussi l'enseignement des écoles françaises comme une appartenance transnationale à la France, et donc à l'Europe, et ainsi comme une protection de leur statut de chrétiens dans un environnement musulman ${ }^{8}$.

Finalement, il ne faut pas oublier que dans la dernière période de l'Empire ottoman, l'influence occidentale, et en particulier française, se révéla considérable dans tous les milieux confessionnels ainsi qu'auprès du gouvernement à Constantinople (Mantran 2003 : 716-719). Cela valait surtout pour le système d'éducation, qui avait été réformé sur le modèle français et où la langue française jouait un rôle important. Il y avait bien sûr également des voix critiques, en particulier des milieux musulmans qui considéraient les écoles françaises comme un danger pour leurs enfants9. Néanmoins, on peut conclure qu'à la fin de l'Empire ottoman, le français avait pu se répandre au Liban en général, et que les catholiques libanais, en particulier, en profitaient.

11 La majorité des écoles françaises étaient entretenues par des ordres religieux, les plus présents au Liban étant les jésuites, les lazaristes, les frères des écoles chrétiennes pour les écoles de garçons; et les filles de la Charité, les sœurs de Saint-Joseph de l'Apparition, les dames de Nazareth pour les écoles de filles ${ }^{10}$. Beaucoup de ces ordres s'étaient établis au Mont Liban où habitait une majorité de chrétiens. Un exemple célèbre est le collège lazariste d'Antoura ${ }^{11}$. Mais il y avait aussi des établissements dans les villes. L'université Saint-Joseph des jésuites, mais aussi les frères des écoles chrétiennes entretenaient des collèges à Beyrouth qui accueillaient chacun environ 1000 élèves. En ce qui concerne le public de ces écoles, Chantal Verdeil, qui a travaillé sur le collège jésuite de Beyrouth, a relevé que malgré une légère ouverture à d'autres milieux, les catholiques composaient la majorité des élèves : en 1900, ils constituaient les trois-quarts des élèves (Verdeil $2007: 28-38,31$ ). Or en 1909, le monopole des écoles françaises catholiques au Liban fut perturbé par l'arrivée de la Mission laïque française à Beyrouth. Cette association, fondée à Paris en 1902, avait pour but de diffuser partout dans le monde un enseignement français laïque (Thévenin 2002 : 20-30). La fondation correspondait en France aux lois qui limitaient ou supprimaient les droits et compétences des ordres religieux, notamment dans le domaine de l'éducation (Ozouf 1982 : 55-92). La Mission laïque critiquait vivement le monopole des congrégations en Orient. Ainsi s'exprime le Bulletin de la Mission laïque en 1906 :

Ce serait à notre avis une erreur dangereuse [...] que de croire que ces deux mots :

Français et catholiques, s'ils ont été longtemps synonymes, doivent le rester 
toujours, et de lier les destinées de l'influence française en Orient aux destinées de l'idée catholique. ${ }^{12}$

De leur côté, les représentants de l'Église catholique percevaient l'école laïque comme un grand danger et allaient jusqu'à menacer les parents. Citons une lettre du délégué apostolique en Syrie de 1911 :

Nous renouvelons, de la manière la plus solennelle, la défense de nos vénérés prédécesseurs de fréquenter les écoles acatholiques. [...] Avec la défense, nous renouvelons la censure ecclésiastique relative, en vertu de laquelle les désobéissants ne pourront être absous et admis aux sacrements sans une autorisation spéciale de Nous. ${ }^{13}$

Le terme " acatholique » employé ici renvoie au deuxième danger perçu par les écoles françaises catholiques, à savoir les écoles protestantes anglaises et allemandes. En effet, celles-ci se multipliaient et étaient très appréciées, en partie d'ailleurs par les maronites ${ }^{14}$. Dans leurs correspondances, les ordres catholiques français n'arrêtaient pas d'y faire référence et de souligner l'importance de les « combattre ». Un lazariste stationné à Beyrouth demandait ainsi à son supérieur à Paris : «Nous avons besoin de lutter contre les protestants, aidez-nous, Bon Père, à le faire. $»^{15}$ Pour cette raison aussi les écoles jésuites donnaient à leurs élèves des devoirs pour l'été: les professeurs voulaient les garder proches d'eux pour qu'ils ne soient pas tentés, dans leurs villages, par des missionnaires anglais ${ }^{16}$. On constate donc une prise en charge totale de l'élève qui se révélait à la fois religieuse et linguistique et donc un mélange des enjeux des deux domaines ${ }^{17}$.

En ce qui concerne la peur des ordres vis-à-vis de la Mission laïque, elle n'était pas justifiée, puisque, au moins dans les premières décennies, les communautés musulmanes, grecques-orthodoxes et juives constituaient les plus grands groupes d'élèves ${ }^{18}$. Cela était moins dû à un rejet des élèves chrétiens de la Mission laïque ${ }^{19}$ qu'au fait que les élèves chrétiens étaient déjà bien servis par les écoles chrétiennes et ne cherchaient pas, comme les familles non catholiques, une école où ils n'aient pas à craindre le prosélytisme ${ }^{20}$.

Bien qu'en France même les ordres religieux aient été de plus en plus privés de leurs droits, leurs subventions au Liban par le gouvernement français restaient intactes et furent même augmentées (Riffier 2000). Le gouvernement ottoman, finalement, acceptait l'ouverture des écoles catholiques françaises, à cause des règlements entre l'Empire ottoman et la France et parce que ces écoles assuraient l'éducation des communautés catholiques ${ }^{21}$. Lors de la Première Guerre mondiale, toutes les écoles étrangères durent néanmoins fermer leurs portes et leurs membres furent obligés de rentrer en France. Ce n'est qu'entre 1918 et 1920 que les écoles françaises purent revenir au Liban, cette fois-ci dans une situation politique bien différente. La France avait obtenu, de la part de la Société des Nations, un mandat sur la Syrie et le Liban. Ce mandat était censé accompagner ces deux pays sur le chemin de l'indépendance. En réalité, la France établissait ou renforçait des structures coloniales (Firro 2003 : p. 9). Ce statut correspondait aux souhaits d'un grand groupe de maronites qui plaidaient, après la chute de l'Empire ottoman, pour un pays à prédominance chrétienne sous la protection de la France ${ }^{22}$. Or, il faut souligner qu'il y avait aussi des catholiques qui plaidaient pour une union du Liban soit avec la Syrie, soit avec d'autres pays arabes (Traboulsi $2007: 80-86$ ). 


\section{La période mandataire : au-delà des frictions, une collaboration commune avec le pouvoir} avec le pouvoir mandataire, ce qui renforça encore leur lien. Ce lien survivait aux différentes malentendus. Avant d'analyser cette relation, il est important de souligner la place centrale des écoles françaises dans la politique mandataire française. Elles constituaient, pour les autorités françaises, le moyen le plus efficace pour atteindre et infiltrer les populations dans les pays sous mandat. Paris augmentait les subventions pour les écoles françaises privées, donc majoritairement catholiques, avant de les baisser à partir de 1923 et de les augmenter de nouveau à partir de 1935, et le HautCommissariat transformait le système éducatif au Liban comme en Syrie en faveur des écoles françaises. Mais c'était aussi l'enseignement privé local, chrétien et musulman, que la France finançait au Liban ${ }^{23}$. Elle pensait pouvoir mieux contrôler ces écoles qu'elle inspectait en même temps qu'elle les subventionnait ${ }^{24}$. Le gouvernement libanais, qui n'avait jusqu'à la fin du mandat que très peu de pouvoir, soutenait cette politique scolaire. On peut donc dire que dans le domaine éducatif, la France procédait comme dans ses colonies où elle veillait à contrôler, par l'éducation, les populations indigènes ${ }^{25}$.

(e mandat, les catholiques libanais continuaient à fréquenter de façon massive les écoles catholiques françaises. Ces écoles correspondaient à leurs convictions religieuses et idéologiques. Le mouvement phénicien, répandu en particulier dans les milieux maronites, est un bon exemple pour cette communauté de pensée transnationale. Les idées sur le peuple phénicien du II ${ }^{\mathrm{e}}$ millénaire avaient été développées par l'historien et philologue français Ernest Renan puis par le jésuite belge Henri Lammens, et avaient par la suite été reprises par le mouvement phénicien autour de l'auteur francophone Charles Corm à la fin de la Première Guerre mondiale. Elles permettaient aux maronites de contourner l'influence arabe et islamique sur le pays et, par l'accent mis sur le cosmopolitisme des Phéniciens, de légitimer l'ouverture sur le monde, à savoir la proximité avec la France (Kaufman 2004). Mais les autres communautés catholiques partageaient la coopération avec le mandat, comme le montrent par exemple les discours tenus lors de la réception officielle du Haut-Commissariat à l'archevêché greccatholique en $1927^{26}$.

De plus, les catholiques investissaient les écoles françaises pour maintenir les positionsclés dans le système administratif et économique mandataire. Les écoles, catholiques en particulier, répondaient à ce désir, parce qu'elles établissaient des liens très proches avec les services du Haut-Commissariat français et avec les entreprises françaises au Liban. Cela se voit par exemple dans la composition des jurys des différents examens passés dans les écoles: à côté des membres libanais, les membres français appartenaient aux administrations, juridictions et commerces français ${ }^{27}$. En effet, les écoles françaises, et en particulier les écoles catholiques, jouaient un rôle médiateur entre le gouvernement mandataire et leurs anciens élèves : en 1920, Béchara al-Khoury fut nommé secrétaire général du gouvernement du Mont Liban sur l'initiative des jésuites (Salibi 1965 : 171). Les familles maronites déjà puissantes en profitaient surtout, mais aussi quelques nouveaux personnages d'autres communautés, comme par exemple Sélim Takla, grec-catholique d'origine, qui devint par la suite ministre des Affaires étrangères (Khoury 2004). Un autre exemple de la possibilité d'intégration des

Documents pour l'histoire du français langue étrangère ou seconde, 45 | 2010 
maronites et d'autres communautés au sein de la société mercantile libanaise est le banquier et lettré Michel Chiha, qui appartenait à la petite communauté chaldéenne au Liban. Il se lia par mariage à la famille Khoury et devint un personnage politique important, notamment dans la construction de la Constitution libanaise (Firro 2003 : 31). Le rôle important que jouaient ces communautés catholiques non maronites pour la diffusion de la langue arabe et pour une intégration de l'Islam et des musulmans dans la pensée catholique libanaise se manifeste de façon exemplaire dans le personnage de Georges Kfoury, directeur des études arabes à la Mission laïque française de Beyrouth. Originaire de Zahlé, et donc probablement de confession grecque-catholique, il fréquentait, à la Mission laïque, surtout les milieux sunnites et grecs-orthodoxes ${ }^{28}$. Les écoles françaises remplissaient donc le rôle de lieux de formation d'élites dans d'autres pays arabes comme la Syrie (Bocquet 2008:14) et l'Égypte (Abécassis $2000: 17$ ). De sorte que le constat de Pierre Bourdieu sur le système scolaire français est aussi valable pour le système scolaire libanais sous le mandat, le rôle de l'école étant, selon lui,

[d']inculquer [...] un rapport à l'institution scolaire et, à travers celui-ci, un rapport au langage et à la culture qui n'est autre que celui des classes dominantes. (Bourdieu \& Passeron 1970 : 159) français n'appuyait plus sa politique mandataire exclusivement sur les catholiques, mais se tournait davantage vers les populations musulmanes. Ce changement de politique était dû, pour une part, au nouveau gouvernement du cartel des gauches en France qui voulait en finir avec la politique catholique au Levant. Une autre raison était la révolte des Druzes en Syrie en 1925 : le gouvernement français prit conscience que les communautés musulmanes risquaient de s'élever contre le mandat et, au pire, de s'unir avec les nationalistes arabes des autres pays sous influence française. Paris décida donc de soutenir les écoles qui accueillaient les musulmans, à savoir les écoles de la Mission laïque ${ }^{30}$. Les écoles catholiques ne manquaient pas de le signaler, de le regretter et de condamner la nouvelle politique française ${ }^{31}$. Certains cercles maronites francophiles le faisaient également, comme en témoignent les articles du journal L'Orient fondé et tenu par d'anciens élèves des écoles catholiques françaises. En 1925, un article formula même l'idée que les musulmans se servaient seulement de la laïcité pour repousser les chrétiens ${ }^{32}$. Ironiquement, il y avait en effet au lycée de la Mission laïque certains parents musulmans qui fréquentaient cette école pour pouvoir percer le monopole de l'enseignement supérieur tenu par les jésuites et dont profitaient surtout les catholiques libanais ${ }^{33}$. Mais il faut aussi souligner qu'à partir de 1925 environ une partie des familles notables musulmanes s'arrangea avec le régime mandataire et commença à coopérer avec les catholiques (Eddé 2001 : 79-102). 

tard : à partir de 1930, les maronites, suivant en cela l'exemple de leur patriarche, exprimèrent leur désaccord avec certaines décisions du Haut-Commissaire français (Zamir 2000 : 192). Comment les écoles françaises réagissaient-elles par rapport à cet éloignement ? Les jésuites se faisaient le porte-voix des maronites mécontents. En 1936, par exemple, le supérieur Sautier rapporte au représentant de l'université Saint-Joseph à Paris, le père Jalabert, son expérience au collège maronite de la Sagesse de Beyrouth, connu pour son nationalisme libanais :

fin décembre, il y avait une pièce à la Sagesse [école fondée par l'archevêque maronite de Beyrouth] pour la fête de Mgr Moubarak; Mgr Boustany y assistait ; à la fin Mgr B. dit quelques mots... il termine: "Vive le Collège de la Sagesse... tonnerre d'applaudissements; "Vive la France... silence glacial; il ajoute alors rapidement : «Vive le Liban...» les applaudissements reprennent très vifs; c'est tout à fait typique. ${ }^{34}$

Sans soutenir ouvertement les maronites, on perçoit que les jésuites regrettaient eux aussi les anciens temps, et rendaient la politique du gouvernement français responsable du sentiment de déception des catholiques libanais. Quelques jours auparavant, Sautier avait noté :

nos amis maronites... sans leur donner raison, je le regrette pour eux et avec eux, car malgré tout, ce sont eux qui ont dans ce pays le cœur le plus français et le plus catholique. ${ }^{35}$

La situation se radicalisa lors des négociations pour un traité franco-libanais en 1936: les positions des différentes tendances politiques au Liban devinrent de plus en plus intransigeantes et revendicatives. Le mécontentement de certains maronites se concrétisa en 1936 dans la création du mouvement Kataeb ou Phalanges libanaises, une organisation de jeunesse qui allait devenir un parti politique et qui représentait la branche la plus radicale du courant politique libanais (Salibi 1965: 181-182). Ce mouvement à dominante maronite s'opposait vivement aux aspirations musulmanes panarabes, mais aussi de plus en plus au mandat français, et luttait par contre pour un Liban indépendant et chrétien (Dueck 2010 : 208). Son fondateur, Pierre Gemayel, ancien élève du collège jésuite de Beyrouth, et son mouvement avaient beaucoup d'adhérents dans cette école. Comment les jésuites y réagissaient-ils? Selon une directive de la fin des années 1930 :

Principe : Pas de politique pour les élèves. Ce n'est pas de leur âge. [...] nuit au travail [...] à la bonne entente. [...] Donc pas d'activité phalangiste officielle au collège. Pas d'insigne [...].

Le personnel enseignant: garde sa liberté : il a l'âge. Pour montrer qu'il n'y a aucune hostilité: port d'insigne autorisé. Mais au contraire sympathie pour beaucoup des buts. ${ }^{36}$

Dans la réalité par contre, selon le témoignage d'un ancien élève de cette école, les Kataeb ont fait de la propagande, de façon discrète, et leurs membres ont participé à des manifestations, avec l'accord des jésuites ${ }^{37}$. De plus, en avril 1937, la revue du collège consacrait un article à "Cinq minutes avec Pierre Gemayel (chef suprême des Phalanges libanaises) $\Perp^{38}$. Malgré un manque d'informations sur les autres écoles catholiques françaises, il est à supposer que l'attitude de leurs responsables était semblable, quoique moins systématique, à propos des Phalanges libanaises.

Documents pour l'histoire du français langue étrangère ou seconde, 45 | 2010 
attitude plus critique envers le mandat français semble aussi avoir attein l'enseignement même. En 1931, le père Honiskiss, du collège jésuite de Beyrouth, rapporte le point de vue d'un élève :

La comparaison [avec d'autres élèves] m'est surtout odieuse, insupportable, lorsqu'elle est faite aux dépens de ma civilisation et de ma culture par quelqu'un qui ne les connaît que superficiellement et qui en juge sur les apparences. Étant par nature apte à m'adapter aux choses les plus variées, capable de m'assimiler ce qu'il y a de bon en elles, j'exige la même attitude de celui qui se mêle de juger ma culture et ma civilisation. ${ }^{39}$

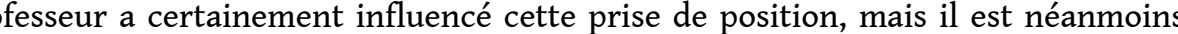
remarquable qu'un tel discours apparaisse au sein de l'école. Il ne faut tout de même pas oublier qu'il y avait d'autres milieux catholiques et même maronites qui ne soutenaient pas Gemayel et qui s'exprimaient explicitement pour une pérennisation du mandat français. Le quotidien francophone maronite de Beyrouth, L'Orient, par exemple, affirmait en 1926 : « la France n'abandonnera pas son mandat $»^{40}$.

Même si les congrégations françaises toléraient ou soutenaient certaines idées politiques maronites critiques envers la France, elles étaient loin d'abandonner l'idée de « mission civilisatrice » à travers la langue et la culture françaises. Au contraire, les écoles catholiques continuaient à louer les bienfaits de cette « mission civilisatrice » et à les enseigner à leurs élèves. Par exemple, le salut au drapeau français était pratiqué. Un autre moyen pour assurer la domination du français était le «signal ». C'était un morceau de bois qui punissait les élèves qui parlaient arabe en cours ou à la récréation. Plusieurs publications ont signalé cet instrument des écoles françaises qui obligeaient les élèves à ne parler que français dans l'établissement ${ }^{41}$. Un ancien élève du collège jésuite de Beyrouth décrit l'obligation du français au détriment de l'arabe comme une "occidentalisation des mœurs", conduisant les élèves libanais à une "rupture avec leur propre milieu, à travers une formation rationalisée qui allait les conditionner " (Mouawad 2005 : 191-210; 200 et 201).

De même, les écoles transmettaient souvent aux élèves une idée orientaliste de leur propre pays. En octobre 1943, un sujet proposé pour le baccalauréat à Beyrouth était le suivant: «L'Orient dans Lamartine. Déterminez en quoi et comment le voyage d'Orient a élargi et surélevé l'inspiration lamartinienne. $»^{42}$ Avec Edward Said, on constate ici une définition de l'Orient par l'Occident que les élèves " orientaux » étaient obligés de reproduire.

, les écoles françaises étaient aussi obligées de reconnaître qu'elles n'œuvraient pas en France, mais au Liban, dont la langue officielle était, à côté du français, l'arabe. Elles comprenaient aussi que le mandat prendrait fin un jour et qu'il fallait donc intégrer la langue et la culture arabes. Curieusement, les mêmes débats qui surgissaient au collège des jésuites au début des années 1940 s'étaient produits au lycée de la Mission laïque vingt ans auparavant. En 1941, par exemple, le compte rendu d'une réunion des professeurs du collège jésuite rapporte : «On aborde le problème des études arabes. [...] Le problème doit être abordé comme un problème d'esprit : l'arabe langue maternelle et non pas langue secondaire. $»^{43}$ Les responsables de la Mission laïque s'étaient déjà confrontés à ce problème en 1922 et le directeur avait conclu : «La langue arabe doit être l'objet de tous nos soins ; [...] les Syriens sont, à juste titre, très chatouilleux sur ce point. $\gg^{44}$ Cette citation indique la raison du décalage entre les deux écoles : les parents des élèves du collège laïque avaient manifesté beaucoup plus tôt un souci pour la langue et la culture arabes que ceux des écoles catholiques. Cela est d'autant plus

Documents pour l'histoire du français langue étrangère ou seconde, 45 | 2010 
frappant que les jésuites eux-mêmes étaient très actifs et influents en ce qui concerne la recherche sur la langue arabe.

Après l'indépendance du Liban en 1943, la situation ne changea que très peu.

\section{Après l'indépendance : continuité, mais ouverture}

Le politologue Theodor Hanf qui a mené, dans les années 1960, une grande enquête sur le système éducatif libanais a conclu que dans ce domaine très peu de choses avaient changé après l'indépendance du pays (Hanf 1969 : 84). Qu'en était-il de la relation des catholiques libanais avec les écoles françaises?

Malgré la déception de certains chrétiens face au retrait de la France du Liban, les liens étaient maintenus. Ces liens étaient toutefois moins exclusifs, s'ouvrant aussi aux musulmans ${ }^{45}$. Tout d'abord, il faut souligner l'étroite relation qui subsistait entre les écoles françaises et leurs anciens élèves qui occupaient après l'indépendance des postes importants dans la vie politique, économique et administrative française. Par exemple, parmi les sept premiers présidents de la République libanaise, obligatoirement des maronites, six étaient d'anciens élèves du collège jésuite de Beyrouth (Mouawad 2005 : 193). Cela veut dire que même si certains milieux catholiques libanais regrettaient, et cela jusqu'à présent, le départ des Français comme pouvoir officiel au Liban, ils continuaient à fréquenter les écoles françaises. La plupart de ces écoles françaises devenaient progressivement libanaises, avec une direction libanaise et un personnel enseignant majoritairement libanais ${ }^{46}$.

Néanmoins, la langue et culture françaises y gardaient une place prépondérante. De même, les gouvernements français successifs veillaient à maintenir un lien avec ces écoles ${ }^{47}$. Le gouvernement libanais, quant à lui, soutenait la continuité du système éducatif mandataire, ce qui signifiait un grand écart entre les écoles privées, qui constituent jusqu'à aujourd'hui des institutions pour les élites du pays, et l'enseignement public, qui recevait et reçoit très peu de moyens.

Même si beaucoup d'écoles catholiques s'ouvraient davantage aux communautés non chrétiennes, d'autres restaient encore très homogènes et ne recevaient presque que des maronites. C'est le cas par exemple du collège Notre-Dame de Jamhour, à une heure de Beyrouth, qui a remplacé l'ancien collège jésuite en ville. Même s'il affirme, dans son projet d'éducation, s'adresser aux "élèves chrétiens et non chrétiens" son public majeur reste catholique, voire maronite.

Curieusement, la Mission laïque s'était par contre ouverte aux chrétiens. Depuis le milieu des années 1930 déjà, son école de Beyrouth avait accueilli davantage de catholiques parmi ses élèves ${ }^{48}$. Cette augmentation correspondait sûrement, d'une part, au fait que l'école accueillait aussi davantage d'élèves français, enfants de fonctionnaires du Haut-Commissariat français. Or, on peut aussi constater, au moins pour la nouvelle période qui commence en 1935, un ancrage de l'école laïque dans le milieu chrétien libanais. Le nouveau directeur, nommé en 1935, Jacques Grandjouan, donnait même des conférences chez les jésuites, que ces derniers appréciaient beaucoup: "Impression d'un homme de très haute culture, causeur extraordinaire (sujet: Pédagogie moderne), et voulant être un homme d'action. » De même, il faisait des efforts pour atteindre aussi les milieux chrétiens, parce que les musulmans devenaient moins nombreux au lycée ${ }^{49}$. Suite à la guerre civile cette tendance s'est 
encore renforcée, parce que la ville a été coupée en deux, et l'école de la Mission laïque était la seule accessible pour beaucoup de parents chrétiens ${ }^{50}$.

En même temps, la relation de concurrence qui avait dominé les écoles françaises pendant longtemps, s'était, depuis l'augmentation de facteurs menaçants de l'intérieur et de l'extérieur, transformée en une relation de coopération ${ }^{51}$.

Aujourd'hui, le rapport entre catholiques libanais et éducation française semble avoir légèrement changé. Même si le français reste pour beaucoup de catholiques libanais leur langue de référence, il semble que ce sont actuellement les milieux musulmans libanais qui recherchent le plus la langue française. Les chrétiens par contre se tournent de plus en plus vers l'anglais. Ce changement est probablement dû au fait que beaucoup de chrétiens libanais ont émigré dans des pays anglophones, en particulier les États-Unis. Une autre raison tient peut-être au changement de la politique libanaise de la France qui n'est plus exclusivement tournée vers les chrétiens du pays des Cèdres. Certains milieux maronites ne ressentent plus la France comme leur protecteur. On pourrait donc émettre l'hypothèse que pour les chrétiens, aujourd'hui minoritaires au Liban, la langue et la culture françaises ont perdu leur «valeur symbolique », et qu'ils cherchent de nouveaux horizons pour exprimer leur statut particulier au Liban et au Proche-Orient.

\section{Conclusion}

L'analyse de la relation des catholiques libanais avec les écoles françaises au Liban à travers les périodes ottomane, mandataire et d'indépendance a permis de relever beaucoup de constantes: les écoles françaises catholiques ont été majoritairement fréquentées par les catholiques libanais; les écoles françaises ont formé et forment encore, aujourd'hui avec les écoles américaines, les élites économiques et politiques du pays; pour beaucoup de maronites la France, et donc la langue et la culture françaises ont été et restent synonymes de christianisme. Or, nous avons aussi vu que ce dernier aspect est en train de changer aujourd'hui.

On peut donc résumer les attitudes et stratégies des catholiques libanais par rapport à la langue française à travers les écoles françaises au Liban de façon suivante : à la fin de l'Empire ottoman, ils s'en sont servis pour affirmer et protéger leur statut de minorité. Sous le mandat français, ils s'en sont servis pour participer à la culture dominante du pays et assurer leur appartenance à l'élite économique, politique et sociale du pays. Après l'indépendance, certains catholiques sont restés dans la nostalgie du français comme bien symbolique des chrétiens, tandis que d'autres se sont orientés vers les pays et l'éducation anglo-saxons.

BIBLIOGRAPHIE 


\section{Bibliographie}

ABÉCASSIS, Frédéric (2000). L'enseignement étranger en Egypte et les élites locales (1920-1960). Francophonie et identités nationales. Thèse de doctorat, université de Provence / Aix-deMarseille.

ABOU, Sélim (1962). Le bilinguisme arabe-français au Liban. Essai d'anthropologie culturelle. Paris : Presses universitaires de France.

AWIT, Henri \& DUCRUET, Jean, s.j. (2006). L'université Saint-Joseph de Beyrouth et ses institutions. Textes fondateurs, dispositions légis-latives et réglementaires et décisions de principe régissant la reconnaissance des diplômes et le régime des équivalences (1875-2002). Beyrouth : Publications de l'université Saint-Joseph.

BOCQUET, Jérôme (2004). Missionnaires français et allemands au Levant : les lazaristes français de Damas et l'Allemagne, du voyage de Guillaume II à l'instauration du mandat, in : Dominique Trimbur (éd.), Europäer in der Levante. Zwischen Politik, Wissenschaft und Religion (19. und 20. Jahrhundert), München : R. Oldenbourg Verlag, p. 57-75.

- (2008). La France, l'Église et le Baas. Un siècle de présence française en Syrie (de 1918 à nos jours). Paris : Les Indes Savantes.

BOURDIEU, Pierre \& PASSERON, Jean-Claude (1970). La repro-duction. Éléments pour une théorie de l'enseignement. Paris : Éditions du Minuit.

CHALABI, Tamara (2006). The Shi' is of Jabal 'Amil and the new Lebanon. Community and nation state 1918-1943. New York : Palgrave Macmillan.

COOPER, Nicola (2004). Making Indo-China French. Promoting the Empire through education, in : Martin Evans (ed.), Empire and culture. The french experience 1830-1940. London : Palgrave Macmillan, p. 131-147.

DUECK, Jennifer (2010). The claims of culture at Empire's end. Syria and Lebanon under french rule. Oxford : Oxford University Press.

EDDÉ, Carla (2001). Étude de la composition du conseil municipal beyrouthin (1918-1953).

Renouvellement des élites urbaines ou consolidation des notables? in : Agnès Favier,

Municipalités et pouvoirs locaux au Liban, Beyrouth : CERMOC, p. 79-102.

FIRRO, Kais (2003). Inventing Lebanon. Nationalism and the state under the mandate. London / New York : I.B. Tauris.

FORTNA, Benjamin (2002). Imperial classroom. Islam, the state, and education in the late Ottoman Empire. Oxford : Oxford University Press.

FOUCAULT, Michel (1975). Surveiller et punir. Naissance de la prison. Paris : Gallimard.

HACHEM, Victor (2003). Antoura, de 1657 à nos jours : une histoire du Liban. Antoura : Presses de Chemaly et Chemaly s.a.l.

- (2008). Le Français et nous. Un humanisme en partage. Mansourieh : Aleph.

HANF, Theodor (1969). Erziehungswesen in Gesellschaft und Politik des Libanon. Bielefeld :

Bertelsmann Verlag.

HEYBERGER, Bernard (2002). Les Européens vus par les Libanais (XVI ${ }^{\mathrm{e}}$-XIX ${ }^{\mathrm{e}}$ siècles), in : Bernard Heyberger \& Carsten Walbiner (éds), Les Européens vus par les Libanais à l'époque ottomane, Würzburg : Ergon Verlag, p. 1-22. 
HUHN, Ingeborg (1989). Der Orientalist Johann Gottfried Wetzstein als preußischer Konsul in Damaskus (1849-1861) dargestellt nach seinen hinterlassenen Papieren. Berlin : Klaus Schwarz Verlag.

JALABERT, Henri (ca 1960). Les institutions scolaires françaises au Liban, in : Georges et Édith Corban-Mertens (éds), France-Liban. Revue, p. 26-29.

- (2001). Histoire d'un siècle 1853-1953. Congrégation des sœurs des Saints-Cœurs de Jésus et de Marie. Beyrouth : Maison centrale.

KASSIR Samir (2003). Histoire de Beyrouth. Paris : Fayard.

KAUFMAN, Asher (2004). Reviving Phoenicia. In search of identity in Lebanon. London / New York : I.B. Tauris.

KHOURY, Gérard D. (1993). La France et l'Orient arabe. Naissance du Liban moderne 1914-1920. Paris : Armand Colin.

KHOURY, Gérard D. (éd.) (2004). Sélim Takla 1895-1945. Une contribution à l'indépendance du Liban. Paris / Beyrouth : Karthala / Dar an-Nahar.

KOUYOUMDJIAN, Ouannès Pacha (2003). Le Liban à la veille et au début de la guerre. Mémoires d'un gouverneur 1913-1915. Paris : Centre d'histoire arménienne contemporaine.

MAKDISI, Ussama (2000). The Culture of sectarianism. Community, history, and violence in nineteenth-century Lebanon. Berkeley et al. : California University Press.

MANTRAN, Robert (2003). Histoire de l'Empire ottoman. Paris : Fayard.

MURAD, Nicolas (1844). Notice historique sur l'origine de la nation maronite et sur ses rapports avec la France, sur la nation druze et sur les diverses populations du Mont Liban. Paris : Libraire d'Adrien Le Clere et Cie.

MOUAWAD, Youssef (2005). L'enfance des chefs. L'éducation au collège secondaire des pères jésuites à Beyrouth (1875-1914), in : Bernard Delpal et al. (éds), France-Levant. De la fin du XVII siècle à la Première Guerre mondiale. Paris : Geuthner, p. 191-210.

MOUAWAD, Joseph (1992). “The Image of France in Maronite Tradition”, Beirut Review, 4, 1992, p. 85-95.

OZOUF, Mona (1982). L'École, l'Église et la République 1871-1914. Paris : Éditions Cana / Jean Offredo. SALIBI, Kamal (1965). The modern history of Lebanon. Delmar / New York : Caravan Books.

- (1988). A house of many mansions. The history of Lebanon reconsidered. Berkeley : University of California Press.

SEIKALY, Samir (2002). Shaykh Yusuf al- Nabahani and the West, in : Bernard Heyberger \& Carsten-Michael Walbiner (éds), Les Européens vus par les Libanais à l'époque ottomane. Würzburg : Ergon Verlag, p. 175-181.

SUERMANN, Harald (2010). Libanon, in : Harald Suermann, Kirche und Katholizismus seit 1945, tome 7, Naher Osten und Nordafrika, Paderborn et al. : Ferdinand Schöningh, p. 105-136.

THEVENIN, André (2002). La Mission laïque à travers son histoire. 1902- 2002. Paris : Mission laïque française.

THOBIE, Jacques (1977). Intérêts et impérialisme français dans l'Empire ottoman, 1895-1914. Paris : Publications de la Sorbonne.

TRABOULSI, Fawwaz (2007). A history of modern Lebanon. London / Ann Arbor : Pluto Press. 
VERDEIL, Chantal (2007). « Un établissement catholique dans la société pluriconfessionelle de la fin de l'Empire ottoman. L'université Saint-Joseph de Beyrouth ", in : Cahiers de la Méditerranée, $\mathrm{n}^{\circ}$ 75 , décembre 2007, Islam et éducation au temps des réformes. Systèmes scolaires et enjeux de l'enseignement au Proche-Orient et en Afrique du Nord aux XIX et XX ${ }^{\mathrm{e}}$ siècles, dossier thématique coordonné par Anne-Laure Dupont, p. 28-38.

ZAMIR, Meir (2000). Lebanon's quest. The road to statehood 1926-1939. London / New York : I.B.

Tauris.

\section{NOTES}

1. Notice historique sur l'origine de la nation maronite et sur ses rapports avec la France, sur la nation druze et sur les diverses populations du Mont Liban, par S. G. Nicolas Murad, archevêque maronite de Laodicée, représentant de sa nation près le Saint Siège, 1844, p. 1. Beaucoup d'historiens ont écrit sur ce texte célèbre, qui construit des liens entre les maronites et la France. Voir par exemple, Mouawad 1992, p. 85-95 ; Firro 2003, p. 15-16.

2. Hachem 2008. Voir aussi la préface à cet ouvrage de l'homme politique Michel Eddé, qui parle d'« une marque d'affection pour la France avec qui les Libanais partagent depuis des siècles les mêmes idéaux de justice, de liberté et de démocratie. »

3. Les premiers ordres catholiques étaient arrivés au XVII ${ }^{\mathrm{e}}$ siècle, mais en nombre très réduit. Voir Heyberger 2002, p. 1-22, p. 6.

4. L'année 1860 constitue une autre étape fondamentale dans la mémoire des catholiques libanais, puisqu'elle marque l'intervention des troupes françaises venues au secours des chrétiens du Mont Liban, en conflit violent avec les Druzes, communauté islamique. Pour beaucoup de chrétiens du Mont Liban, cette intervention marqua le début et / ou la continuité du soutien de la France. Voir Makdisi 2000, p. 138. Alors que les sources françaises et libanaises chrétiennes célèbrent ce geste français, un diplomate allemand à Damas à l'époque rapporte très sèchement que les Français étaient arrivés trop tard. Voir Huhn 1989, p. 230.

5. Comme l'a montré Jacques Thobie, la majorité de l'économie ottomane était à la fin du XIX et au début du XXe siècle entre des mains françaises. Voir Thobie 1977.

6. Voir par exemple les Archives de la Mission laïque française / Paris, 60 AJ 120, lettre de deux professeurs d'Adana au directeur, Adana / 09.10.1912.

7. Voir Archives des jésuites / Beyrouth, 12.C.10, Tanaïl I. Ministères Écoles, lettre en arabe de Salîm Nasîf aux jésuites, novembre 1910.

8. Voici ce qu'écrit un père au Consul général français à Beyrouth en 1903 : «Parmi ses [de la France] hauts faits et ses actes humanitaires, nous nous plaisons surtout à en rappeler un qui a pour résultat le bien moral ainsi que l'inculcation de l'amour de cette France. » Archives du MAE / Nantes, Beyrouth Consulat général Série A, 191 : demandes de bourses, lettre de Elie R. Rizcallah au Consul général, 15.07.1903.

9. Voir par exemple Seikaly 2002, p. 175-181.

10. Les jésuites avaient aussi fondé un ordre libanais proche de leur ordre, les sœurs des SaintsCœurs. Voir Jalabert 2001.

11. Voir par exemple Hachem 2003.

12. Archives de la Mission laïque française / Paris, Bulletin de la Mission laïque française, 1906, p. 66-67.

13. Lettre pastorale au clergé et au peuple latin du vicariat apostolique d'Alep pour le carême de 1911: «Religion et laïcisme en face du problème de l'éducation populaire ", Beyrouth: Imprimerie Catholique, 1911, p. 32-33. 
14. Voir Archives des lazaristes / Paris, Tripoli Correspondance 1892-1913, Lettre de Thomas, Tripoli / 27.03.1907.

15. Archives des lazaristes / Paris, Beyrouth Correspondance 1896-1918, Lettre de Houan, Beyrouth / 16.01.1910.

16. Voir Archives des jésuites / Beyrouth, Tanaïl. École normale. Bulletin de nouvelles, Lettres du Père Degivry (1864-1949) aux « Chers normaliens » pendant les vacances d'été.

17. Cet épisode montre que l'appartenance nationaliste commençait à jouer un plus grand rôle et se confondait chez les ordres religieux avec les hostilités reli-gieuses. Cela était aussi vrai pour la composition des ordres, comme Jérôme Bocquet l'a vérifié pour l'ordre des lazaristes à Damas : la répartitition interna-tionale des Pères allait se dissoudre au début du vingtième siècle en faveur d'une majorité de Pères français et plus nationalistes. Voir Bocquet 2004, p. 57-75.

18. Voir les statistiques dans le fonds d'archives de la Mission laïque française à Beyrouth, in : Archives nationales / Paris, 60 AJ 118-140.

19. Au contraire, le secrétaire général rapporta en 1913 avoir fait de la propagande auprès des familles musulmanes et chrétiennes de la ville. Voir Archives nationales / Paris, 60 AJ 119, lettre de Besnard d'Alexandrie, 04.01.1913. Et en 1925, le secrétaire général de la Mission laïque racontait que dans le passé on avait, pour satisfaire les plaintes des familles chrétiennes, remplacé les professeurs d'arabe musulmans par des maîtres chrétiens. Voir Archives nationales / Paris, 60 AJ 128, lettre de Besnard à Ruche, 09.11.1925.

20. Une autre raison qui a pu empêcher l'arrivée des élèves catholiques à la Mission laïque était sa réputation franc-maçonne.

21. Au Mont Liban, les gouverneurs les accueillaient encore plus volontairement. Le dernier mutasarif du Mont Liban entre 1913 et 1915, Ouannès Pacha Kouyoumdjian, par exemple, malgré sa critique du consul français, loue dans ces mémoires «l'œuvre civilisatrice des nombreux établissements d'éducation entre-tenus et patronnés par la France.» (2003, p. 21). On constate aussi un certain mépris pour les habitants du Mont Liban lorsqu'il parle de « ces peuples à peine sortis de l'état barbare ». Voir ibid., p. 22.

22. Pour les différents courants politiques au Liban à la fin de la Première Guerre mondiale, voir Traboulsi 2007, p. 80-86. Bien que le gouvernement français ait entendu et respecté les souhaits des maronites, il n'y avait répondu qu'en partie: certes, le nouveau Liban avait une légère majorité chrétienne, mais il avait intégré plus de parties de l'ancien Empire ottoman que les catholiques libanais ne l'avaient souhaité. De sorte qu'on a pu considérer que Paris avait cherché à s'assurer la loyauté catholique au Liban, mais avait fait prévaloir ses intérêts impériaux dans cette région cruciale du point de vue géopolitique. Voir Khoury 1993, p. 396.

23. Contrairement à la Syrie, où Paris investissait aussi dans l'enseignement public, les autorités françaises déclaraient qu'au Liban les écoles officielles étaient dans un état tellement lamentable que cela n'en valait pas la peine. Voir Archives nationales / Beyrouth, Recueil des Actes administratifs du Haut-Commissariat de la République Française en Syrie et et au Liban, tome 1-4, 1919-1923, Arrêté 1107 « concernant les écoles privées ».

24. Cette politique s'explique par le fait qu'au Liban, l'enseignement privé était surtout fréquenté par les chrétiens, c'est-à-dire les communautés que Paris voulait satisfaire en premier. Voir Hanf 1969, p. 83-84 : pendant la période mandataire, 49,4\% des élèves scolarisés au Liban fréquentaient des écoles catholiques.

25. Pour les parallèles avec l'Indochine, voir Cooper 2004, p. 131-147, p. 137.

26. Voir Archives L'Orient Le Jour / Beyrouth, 20.04.1927, p. 1.

27. Voir Archives du collège français du Sacré-Cœur / Beyrouth, Palmarès, 1923-1924, p. 27.

28. Archives de la Mission laïque française / Paris, 60 AJ 124, «Rapports mensuels ", novembre 1922. Avant Georges Kfoury, deux autres professeurs de Zahlé portant le même nom de famille, Asaf Bey Kfoury et Paul Kfoury, avaient enseigné dans cette école. Il semble donc que la Mission laïque avait recruté ses professeurs d'arabe dans la même communauté. 
29. Archives des lazaristes / Paris, Association Amicale des Anciens Élèves d'Antoura, bulletin annuel de 1938. Il est intéressant de constater que les anciens élèves des écoles catholiques françaises restaient beaucoup plus attachés à leurs écoles que ceux de la Mission laïque française, ce dont le directeur de l'école de la Mission laïque ne cessait de se plaindre : « Il faut faire un effort pour retenir nos anciens élèves et les empêcher d'aller grossir les rangs de la jeunesse catholique ou protestante. » Archives nationales / Paris, 60 AJ 135, Rapport mensuel du 20 mai au 20 juin 1933. Pourquoi ces attitudes différentes? À mon avis, elles s'expliquent par le fait que les élèves de la Mission laïque avaient fréquenté leur école principalement pour des raisons pragmatiques / rationnelles et de prestige, tandis que les élèves des écoles catholiques étaient liés à leurs écoles aussi par leur confession.

30. Le Haut-Commissariat se chargea donc « d'établir des relations étroites entre l'enseignement donné dans les écoles laïques et l'enseignement religieux ». Archives des jésuites / Beyrouth, 8.C. 21: Haut-Commissariat français: questions scolaires, Rapport concernant la réunion des conseillers et inspecteurs de l'Instruction publique.

31. En 1935, par exemple, le père jésuite Jalabert se plaignait de la manière suivante : « Beaucoup plus négligées naguère, les œuvres laïques (Missions laïques) jouissent d'une situation privilégiée et recueillent la majeure partie de la manne officielle. Surtout, la diffusion de l'esprit laïque, l'excès des libertés (de pensée, de presse), la liberté de nos mœurs, portent atteinte à l'esprit religieux qui a toujours été, et qui heureusement demeure encore, une des nobles caractéristiques des peuples d'Orient. » Archives des jésuites / Vanves, Fonds Jalabert, 1 / 1 : A-K, Deux conférences de L. Jalabert à l'université catholique du Louvain les 10 et 11 décembre 1935.

32. Voir Archives L'Orient Le Jour / Beyrouth, 25.03.1925, p. 2 : « Les éloges du laïc ».

33. Voir les comptes rendus du comité de patronage de la Mission laïque française, par exemple Archives nationales / Paris, 60 AJ 127, séance du 11.11.1925.

34. Archives des jésuites / Paris, Fonds Jalabert, 26-L : Correspondance Sautier-Jalabert, lettre de Sautier à Jalabert, 24.01.1936.

35. Archives des jésuites / Paris, Fonds Jalabert, 26-L : Correspondance Sautier-Jalabert, lettre de Sautier à Jalabert, 21.01.1936.

36. Archives des jésuites / Beyrouth, 11.D.14 : Beyrouth U.S.J. Collège Secondaire III.

37. Voir l'entretien de l'auteur avec Camille Hechaïme, Beyrouth / 24.10.2008.

38. Archives du collège Notre-Dame de Jamhour / Liban, « Nous du Collège. Gazette du Collège U.S.J. Beyrouth ", n 7, avril 1938, p. 4.

39. Archives des jésuites / Vanves, RPO, 62, «L'Enseignement à l'université Saint-Joseph. Le point de vue de l'élève. 30.10.1931. P. J. Honiskiss».

40. Archives L'Orient Le Jour / Beyrouth, 24.07.1926, p. 1.

41. Bocquet 2008, p. 61. Michel Foucault dans son ouvrage Surveiller et punir en parle déjà comme d'un moyen apportant "la technique de commandement et la morale de l'obéissance " des frères des écoles chrétiennes en France au XIX ${ }^{e}$ siècle (Foucault 1975, p. 195). Alors que beaucoup de professeurs dans les écoles françaises le louaient et le défendaient jusqu'à nos jours, il y avait aussi, au sein de la Mission laïque, des discussions autour de son utilité.

42. Archives du collège Saint-Joseph, Antoura / Liban, « Cahier de sujets de devoirs français ».

43. Archives des jésuites / Beyrouth, 11.D.14 : Beyrouth U.S.J. Collège secondaire III, Réunion du 10 février 1942.

44. Archives nationales / Paris $60 \mathrm{AJ}$ 125, lettre de Mathieu à Besnard, Beyrouth / 15.11.1922. Dans le même sens, les écoles catholiques se réunissaient en 1941 autour de la "Semaine sociale » pour réfléchir sur l'adaptation des programmes scolaires aux besoins du pays. Voir Deuxième semaine sociale de Beyrouth. L'École et l'éducation nationale, 27 avril - 3 mai 1941, Beyrouth : Imprimerie Catholique.

45. Voir pour les chiites : Chalabi 2006, p. 150. 
46. Certains des anciens professeurs regrettaient eux aussi le départ des Français et se souviennent de l'époque du mandat comme d'un âge d'or, où le niveau était brillant et où tous les élèves parlaient un français parfait. C'est le cas, par exemple, du frère Michel, ancien professeur au collège du Sacré-Cœur des frères des écoles chrétiennes.

47. Voir par exemple les Archives du collège Notre-Dame de Jamhour, dossier «Coopérations culturelles ».

48. Voir les statistiques in : Archives nationales / Paris, 60 AJ 118-140.

49. Voir Archives nationales / Paris, 60 AJ 138, lettre de Grandjouan à Besnard, Beyrouth / 05.02.1936 : « Le goût de l'instruction chez les Musulmans a causé la création et le développement d'écoles musulmanes à Beyrouth ; ces écoles nous enlèvent notre clientèle (de garçons surtout) ; les Musulmans, qui étaient peut-être 600 sur 800 (inscrits!) il y a dix ans, sont maintenant 240 sur 700 (environ). Situation nouvelle, qui doit peut-être dicter une attitude nouvelle. »

50. Voir entretien de l'auteur avec Souad Slim, Beyrouth / 06.12. 2008.

51. Voir par exemple Jalabert ca 1960 , p. 26-29, p. 28 : "Après une première période assez pénible, les rapports ne tardèrent pas à se normaliser entre les maisons installées depuis longtemps et les nouveaux venus, pour aboutir enfin à la grande cordialité qui est de règle aujourd'hui. »

\section{RÉSUMÉS}

Cette contribution traite de la relation complexe entre catholiques libanais et écoles françaises au Liban entre 1900 et 1950. Elle a pour but de montrer que cette relation a une longue histoire et que sous l'influence grandissante de la France au Liban elle s'est encore consolidée parce qu'elle servait la quête d'identité politique et sociale des deux groupes. Alors que la période du régime ottoman témoignait d'un soutien et besoin réciproques entre catholiques libanais et écoles françaises, celle du régime mandataire fut marquée par un élargissement de cette coopération au profit d'un établissement des deux groupes au sein de l'élite politique, économique et sociale du pays. La période d'indépendance vit finalement la poursuite de cette relation étroite, mais aussi une plus grande ouverture vers d'autres communautés du pays, en particulier les sunnites. On peut donc parler d'un partage d'intérêts idéologiques mais aussi pragmatiques qui a survécu aux difficultés affrontées.

This contribution deals with the complex relationship between the Lebanese Catholics and the French schools in Lebanon between 1900 and 1950. The intention here is to show that this relationship goes back to a long common history and was reinforced under the growing influence of France in Lebanon given that the cooperation helped both groups to find their political and social identity. The period of the late Ottoman Empire witnessed a close relationship of reciprocal need and support, the Mandate regime let both groups seek and find their place in the core of the political, economic and social elite positions of the country. After independence, the narrow relationship between Lebanese Catholics and French schools was pursued, but also broadened towards other communities in the country. One can thus speak of a common ground of ideological, but also pragmatic reasons which persisted despite difficulties. 
INDEX

Keywords : Lebanese catholics, Maronites, Greek-Catholics, French schools, Lebanon, linguistic and social identity, Jesuits, Lazarists, Brothers of the Christian Schools, Mission laïque française Mots-clés : catholiques libanais, Maronites, Grecs-catholiques, écoles françaises, Liban, identité linguistique et sociale, Jésuites, Lazaristes, Frères des écoles chrétiennes, Mission laïque française

\section{AUTEUR}

ESTHER MOELLER

Institut für europäische Geschichte, Mayence,Allemagne

moeller@ieg-mainz.de 